\title{
WHAT CAN WE LEARN FROM THE LOCAL GROUP ABOUT THE ROLE OF INTERACTIONS IN GALAXY FORMATION
}

\author{
K.C. FREEMAN \\ Mount Stromlo and Siding Spring Observatories \\ The Australian National University \\ Canberra, AUSTRALIA
}

\section{Introduction}

This talk is mainly about the halos and bulges of Local Group galaxies, in particular those of the Milky Way and M31, and what they can tell us about the role of interactions in galaxy formation. Bulges are potentially of particular interest for galaxy formation because they are widely suspected to be the seeds for galaxy formation. Sites of active star formation at high redshift are often regarded as spheroids in the process of formation. In this talk, I will say little about disks because their formation is conceptually understood, although many details remain uncertain. To form such flat systems, a fairly undisturbed dissipative process is needed. This process occurred fairly early: the disks appear to be in place by a redshift $z=1$ (Ellis 1997), with a distribution of scalelengths that is similar to the distribution at $z=0$.

\section{The Milky Way}

The Milky Way has two non-disk luminous components: the metal-poor stellar halo and the bulge. Interactions and accretion of small satellite systems may be a very important element in the formation of the halo. On the other hand, the bulge may tell us little about the interaction history of the Milky Way. 


\subsection{THE METAL-POOR HALO}

This component, which includes the metal-poor globular clusters and field stars, has a mass of only about $1 \times 10^{9} M_{\odot}$. Its stars have a heavy element abundance $[\mathrm{Fe} / \mathrm{H}]<-1$. It is kinematically hot and slowly rotating, and is probably unrelated to the bulge: the kinematics of the halo stars $([\mathrm{Fe} / \mathrm{H}]<$ $-1)$ and the more metal-rich bulge stars are very different in the region where they overlap (eg. Morrison \& Harding 1993). This slowly rotating (maybe even retrograde: Majewski 1992) subsystem in an otherwise rapidly rotating galaxy requires explanation. It is now fairly widely believed that the metal-poor halo comes mainly from the accretion of small metal-poor dwarf galaxies during and after disk formation, as suggested by Searle and Zinn (1978). The evidence includes:

- the weak dependence of the kinematics of halo stars on metallicity (eg. Beers \& Sommer-Larsen 1995). A stronger dependence would be expected in a more monolithic dissipative picture.

- the anisotropy of the velocity ellipsoid for halo stars changes with radius, from radial anisotropy in the inner halo to tangential anisotropy in the outer halo (Sommer-Larsen et al. 1994). This can be readily understood from the effects of dynamical friction and tidal disruption on small dwarf galaxies (eg. Quinn \& Goodman 1986).

- the presence of moving stellar groups (eg. Eggen 1979) and stellar streams (Majewski 1994, Lynden-Bell \& Lynden-Bell 1995) in the halo. These groups and streams presumably represent the debris of accreted and disrupted satellites.

- on-going accretion: the apparently disrupting Sgr dwarf (Ibata et al. 1994) is a very direct example. The young metal-poor stars discovered by Preston et al. (1994) are another example. These stars are typically older than about $3 \mathrm{Gyr}$, have abundances $[\mathrm{Fe} / \mathrm{H}]<-1$, a velocity dispersion of about $90 \mathrm{~km} \mathrm{~s}^{-1}$ and rotation of about $130 \mathrm{~km} \mathrm{~s}^{-1}$ (ie. intermediate between the halo and the disk). These stars contribute about $10 \%$ of the local halo density, and could come from the accretion of Carina-like dwarf spheroidal systems with intermediate-age populations. The total associated accreted mass is about $10^{8} M_{\odot}$.

In summary, the process of halo-building by accretion is a continuing process in the Milky Way, not something that happened only long ago.

\subsection{THE BULGE OF THE MILKY WAY}

Although the origin of bulges is an interesting and important problem, and may in some cases be relevant to the formation of the parent galaxies, it is clear that bulges are not an essential element of galaxy formation, 
because many disk galaxies do not have bulges. In the Milky Way, the boxy bulge is barlike. Long ago, de Vaucouleurs (1964) classified our Galaxy as $\mathrm{SAB}(\mathrm{rs}) \mathrm{bc}$ from a range of morphological arguments, and Gerhard (1997) has summarized the detailed recent evidence for the galactic bulge/bar. In brief, the evidence includes:

- the distribution and kinematics of bulge stars (the $C O B E$ /DIRBE light distribution, clump stars, OH/IR stars, planetary nebulae ...).

- the gas distribution and kinematics in the inner Galaxy.

- the high optical depth for microlensing towards the galactic bulge.

$\mathrm{N}$-body simulations of self-gravitating disks strongly suggest that most of the small boxy/peanut bulges, like the bulge of the Milky Way, are bars arising from planar and vertical instabilities of disks (eg. Combes et al. 1990; Pfenniger \& Friedli 1991). Some of these structures may themselves be triggered by interactions (eg. Noguchi 1987). Until now, observational verification of the bar-like nature of boxy bulges has been difficult, because boxy bulges are seen most clearly in edge-on spirals, and then it is not so clear whether they are barred. Recently Kuijken \& Merrifield (1995) have devised a kinematical test of the bar-like nature of near-edgeon boxy/peanut bulges. This test, which depends on the properties of the two principal orbit families in the gravitational field of a rotating bar, is particularly effective and direct for galaxies with extended emission lines in the region of the bulge. Bureau (1997) has applied the Kuijken-Merrifield test to 19 edge-on boxy/peanut bulges and a non-boxy control sample. He found that 15 of the 19 have extended emission lines, so could show the Kuijken-Merrifield effect. Of these 15, 11 show the effect clearly, 3 are very dusty so the effect may be masked, and one galaxy is disturbed by interaction. None of the 7 galaxies in the non-boxy control sample shows the effect. One can conclude from his work that most boxy/peanut bulges are indeed bar-like.

If these boxy bar/bulges arise from instabilities of an equilibrium disk or a forming disk, then they are just consequences of disk formation and dynamics, and are not themselves the seeds of galaxy formation. If this is true, then they are probably not very relevant to understanding interactions in galaxy formation. (The small boxy bulges are certainly interesting in other contexts. I have skipped over some striking properties of these small bulges, such as their exponential structure (not $r^{1 / 4}$, Courteau et al. 1996) and the similar exponential scale heights $(\simeq 300 \mathrm{pc})$ of the Milky Way's disk and bulge.) 


\section{The Bulge of M31}

M31 has a real $r^{1 / 4}$ bulge, from a radius of $200 \mathrm{pc}$ out to $20 \mathrm{kpc}$ (Pritchet $\&$ van den Bergh 1994). Its bulge is flattened, with an axial ratio of about 0.6 (E4).

The chemical properties of the outer bulge or halo of M31 are very different from the halo of the Milky Way. The mean abundance $[\mathrm{Fe} / \mathrm{H}]$ $\simeq-0.6$, much higher than for the Milky Way. The outer bulge shows a large spread in chemical abundance, from about $[\mathrm{Fe} / \mathrm{H}]=-2$ to $-0.2(\mathrm{eg}$. Durrell et al. 1994, Couture et al. 1995, Rich et al. 1996, Holland et al. 1996), with little or no abundance gradient out to a radius of $40 \mathrm{kpc}$.

I suspect that the prominent, more metal-rich bulge dominates the metal-poor population at all radii, as it does in the giant ellipticals. Is it just a semantic point, whether one calls this "outer bulge" a bulge or a halo? I think not, and most of the rest of this talk will be used to argue why not.

\section{The Structure and Chemical Properties of Large Bulges}

These large bulges, as in M31, M104 (the Sombrero galaxy) and NGC 7814, follow the $r^{1 / 4}$ surface brightness distribution. An $r^{1 / 4}$ light distribution is usually associated with a fairly violent merger or aggregation history (eg. van Albada and van Gorkom 1977, Barnes 1988). Chemically, the bulges of spirals show a $(\mathrm{Mg} / \mathrm{Fe})$ - absolute magnitude relation in the same sense as for ellipticals: the brighter bulges show a more marked overabundance of $\mathrm{Mg}$ relative to $\mathrm{Fe}$ (Jablonka et al. 1996). The usual interpretation of this effect is that, after the first major burst of star formation, supernova-driven winds in the more luminous systems remove the remaining gas quickly, and so reduce the subsequent Fe-enrichment by the slower SN of type I. If this is all correct, then it implies that the formation of large bulges occurred early and quickly, much as for giant ellipticals.

\section{The Rotation of Bulges and Ellipticals}

Bulges lie close to the oblate isotropic rotator curve in the $(V / \sigma-\epsilon)$ plane while, from the kinematics of their inner regions $\left(r \lesssim r_{\mathrm{e}}\right)$, the giant ellipticals mostly lie well below the oblate curve. This suggested that the giant ellipticals have lower specific angular momentum $J / M$ relative to the spirals (see Fall, 1983). The apparent difference in specific angular momentum was a long-standing puzzle, because cosmological simulations give similar distributions of $J / M$ in high density and low density regions of the universe, which are usually associated with ellipticals and spirals respectively. So what has happened to the angular momentum of the giant ellipticals? 


\section{The Outer Regions of Giant Ellipticals}

Planetary nebulae can be used to study the kinematics of the outer regions of giant ellipticals $(r \gtrsim 20 \mathrm{kpc})$, well beyond the radius accessible to integrated light spectroscopy. Three giant ellipticals have so far been studied: Cen A (Hui et al. 1995), NGC 1399 (the cD galaxy in the Fornax cluster; Arnaboldi et al. 1994) and NGC 1316 (a large late-merger system in the Fornax cluster; Arnaboldi et al. 1998). In all of these systems, the planetary nebulae studies show that the outer regions are relatively rapidly rotating. When this outer angular momentum is included, the specific angular momenta of the giant ellipticals does indeed appear to be similar to that of the spirals, as predicted by the simulations. Most of the angular momentum of these giant ellipticals resides in their outer regions.

We might expect some morphological evidence of rapid rotation in the outer regions of giant ellipticals. This is seen. The giant elliptical M87 in the Virgo cluster appears as an almost round E0 to E1 system on sky survey images. However, a very deep image of M87, prepared by D. Malin, shows that the outer regions are much more elliptical, about E4. A similar effect was seen by Porter et al. (1991) in a photometric study of brightest cluster ellipticals. They found that the mean ellipticity of these galaxies increases strongly with radius, as we would expect if their outer regions were rotating more rapidly.

Why does the angular momentum of the giant ellipticals reside in their outer regions? This kind of angular momentum segregation is seen in simulations of hierarchical galaxy formation (eg. Quinn et al. 1988), and in the outcome of major and multiple mergers (eg. Weil \& Hernquist 1996). The angular momentum is transported outwards by torques generated while the system is out of equilibrium. If we accept this segregation of angular momentum as indicating a history of hierarchical aggregation or multiple mergers, then the internal angular momentum distribution in large bulges (like those of M31 and M104) gives a pointer to their formation history.

At this time, data on the kinematics of the outer regions of bulges is available only for M104, from unpublished planetary nebulae observations (Freeman et al. 1998). Even for this system the data reach out to only moderately large radii $(\simeq 12 \mathrm{kpc}$ ). From previous work (Kormendy \& Illingworth 1982, Jarvis and Freeman 1986), it was already known that the inner regions of M104 lie close to the oblate isotropic rotator curve in the $(V / \sigma-\epsilon)$ plane, unlike the giant ellipticals. The velocity dispersion is roughly constant with radius, at about $200 \mathrm{~km} \mathrm{~s}^{-1}$. The mean stellar rotational velocity of the bulge is about $100 \mathrm{~km} \mathrm{~s}^{-1}$ near the equatorial plane and remains constant with radius in the outer regions. Comparing the kinematics of M104 with those of the giant ellipticals NGC 1399 and 
NGC 1316, we note that the ellipticals have slow rotation in the inner regions (unlike M104) but the rotation in their outer regions is again about half of the velocity dispersion, as in M104.

If angular momentum redistribution has occurred in M104, then it was clearly not as extreme as in the giant ellipticals, despite the very well established structural $r^{1 / 4}$ law. It would be interesting to know the properties of the angular momentum distribution in the outer bulge of M31: this would not be difficult to measure.

Why do the inner regions of large bulges rotate rapidly, while the inner regions of giant ellipticals do not? What is the essential difference in their formation histories? (We note again that the total specific angular momenta for large spirals and large ellipticals appear to be fairly similar).

One possibility is that bulge formation went on in the presence of a substantial envelope of high angular momentum gas, which later dissipated to form the disk. Some of this gas may have funneled into the inner bulge, through the torques that redistribute angular momentum, and so produced a more rapidly rotating inner bulge. This envelope of gas is presumably absent in the formation of the diskless giant ellipticals.

\section{Summary}

- the halo of the Milky Way continues to be built by the accretion of dwarf galaxies.

- small boxy bulges like that of the Milky Way are mostly barlike, probably grow from the disk, and probably tell us little about the interaction history of their parent galaxies.

- the angular momentum distribution within large bulges, out to large radii, is a useful clue to their merger/aggregation history. M31 is important in this context because the kinematics of its outer bulge are relatively easy to study.

- The difference between outer stellar halos (as in the Milky Way) and outer bulges (as in M31 and M104) may have to do with the answer to the following question:

did the stars that now inhabit the outer regions form together with the aggregation of the dark corona, so that the redistribution of angular momentum also affected the stellar system

\section{OR}

did the stellar bulge/halo form later by accretion, so was not affected by this angular momentum redistribution? 


\section{References}

Arnaboldi, M. et al. 1994. ESO Messenger, June 1994, p 40.

Arnaboldi, M. et al. 1998. In preparation.

Barnes, J. 1988. Astrophys.J., 331, 699.

Beers, T. \& Sommer-Larsen, J. 1995. Astrophys.J.Suppl., 95, 175.

Bureau, M. 1997. Poster paper at this symposium.

Combes, F. et al. 1990. Astron.Astrophys., 233, 82.

Courteau, S. et al. 1996. Astrophys.J., 457, L73.

Couture, J. et al. 1995. Astron.J., 109, 2050.

de Vaucouleurs, G. 1964. In "The Galaxy and the Magellanic Clouds", ed F. Kerr \& A.

Rodgers (Sydney: Aust. Acad. Sci), p 88.

Durrell, P. et al. 1994. Astron.J., 108, 2114.

Ellis, R.S. 1997. Talk presented at IAU Symposium 183.

Eggen, O.J. 1979. Astrophys.J., 229, 158.

Fall, S.M. 1983. In "Internal Kinematics and Dynamics of Galaxies", ed E. Athanassoula

(Dordrecht: Reidel), p 391.

Freeman, K.C. et al. 1998. In preparation.

Gerhard, O. 1997. Talk presented at IAU Symposium 184.

Holland, S. et al. 1996. Astron.J., 112, 1035.

Hui, X. et al. 1995. Astrophys.J., 449, 592.

Ibata, R. et al. 1994. Nature, 370, 194.

Jablonka, P. et al. 1996. Astron.J., 112, 1415.

Jarvis, B. \& Freeman, K.C. 1986. Astrophys.J., 295, 324.

Kormendy, J. \& Illingworth, G. 1982. Astrophys.J., 256, 460.

Kuijken, K. \& Merrifield, M. 1995. Astrophys.J., 443, 13.

Lynden-Bell, D. \& R. 1995. Mon.Not.R.Astron.Soc., 275, 429.

Majewski, S. 1992. Astrophys.J.Suppl., 78, 87.

Majewski, S. 1994. Astrophys.J., 431, L17.

Morrison, H.L \& Harding, P.X. 1993. Publ.Astron.Soc.Pac., 105, 977.

Noguchi, M. 1987. Mon.Not.R.Astron.Soc., 228, 635.

Pfenniger, D. \& Friedli, D. 1991. Astron.Astrophys., 252, 75.

Porter, A. et al. 1991. Astron.J., 101, 1561.

Preston, G. et al. 1994. Astron.J., 108, 538.

Pritchet, C. \& van den Bergh, S. 1994. Astron.J., 107, 1730.

Quinn, P. \& Goodman, J. 1986. Astrophys.J., 309, 472.

Quinn, P. \& Zurek, W. 1988. Astrophys.J., 331, 1.

Rich, R.M. et al. 1996. In "Formation of the Galactic Halo ... Inside and Out", ed H. Morrison and A. Sarajedini (San Francisco: ASP), p 544.

Searle, L. \& Zinn, R. 1978. Astrophys.J., , 225, 357.

Sommer-Larsen, J. et al. 1994. Mon.Not.R.Astron.Soc., 271, 743.

van Albada, T.S. \& van Gorkom, J. 1977. Astron.Astrophys., 54, 121.

Weil, M. \& Hernquist, L. 1996. Astrophys.J., 460, 101. 